\title{
Robust Swing Leg Placement under Large Disturbances
}

\author{
Ruta Desai and Hartmut Geyer
}

\begin{abstract}
Swing leg placement is vital to dynamic stability in legged robots and animals. The most common approaches to generating swing leg motions in robotics use either position or impedance tracking of defined joint trajectories. While these approaches suffice in humanoids, they severely limit swing leg placement under large disturbances in prosthetic limbs, for which stabilizing reactions cannot be planned centrally. Rather than careful central planning, animals and humans seem to rely on local feedback control for reliable swing leg placement. Motivated by this observation, we here present an alternative for generating swing leg motions. We develop a local swing leg control that takes advantage of segment interactions to achieve robust leg placement under large disturbances while generating trajectories and joint torque patterns similar to those patterns observed in human walking and running. The results suggest the identified control as a powerful alternative to existing swing leg controls in humanoid and rehabilitation robotics.
\end{abstract}

\section{INTRODUCTION}

Swing leg placement is vital to stability in legged robots and animals. Without placing the feet into proper target points on the ground, legged systems fail to balance dynamically [1]. These targets have been identified with simplified point-mass models including the linear inverted pendulum for standing and walking [2], [3], and the spring-mass system for running [4], [5]. The models predict foot placement targets which stabilize gait in response to a disturbance such as an external push or an unexpected change in the ground level [3], [6][9]. However, they do not reveal how the segmented legs of humans and humanoids can actually reach these targets.

The most common approach to generating swing leg motions in humanoids uses trajectory planning and tracking. In this approach, trajectories for all leg joints are planned either based on optimization over deviations from the foot placement targets [10] or by spline interpolation between consecutive placement targets [11], [12]. Once generated, these reference trajectories are tracked via proportionalderivative control at the joint levels. Planning and tracking of swing-leg trajectories requires central control over all leg joints, which limits the application of this approach to powered prosthetic limbs which replace only part of the human body.

Although alternative approaches have been explored in rehabilitation robotics [13], [14], tracking predefined joint patterns remains the state of the art in the control of powered artificial legs [15]-[17]. For instance, current leg prostheses mimic human joint impedances that have been recorded in experiments [16]. Bound to these predefined

R. Desai and H. Geyer are with the Robotics Institute, Carnegie Mellon University, 5000 Forbes Avenue, Pittsburgh, PA 15213, USA. \{rutad, hgeyer\}@cmu.edu patterns, handling gait disturbances still requires event detection, automatic classification, and the implementation of deliberate joint trajectories to counter these disturbances [18], hampering practical implementations of autonomous powered legs that achieve robust swing placement under large disturbances.

Animal and human legs by contrast demonstrate robust swing placement with remarkable autonomy. From early work on mesencephalic cats [19] to recent investigations on paralyzed cats and rats with drug administration and epidural stimulation [20], experiments have shown that animals can seamlessly adapt their leg behavior throughout stance and swing, to different speeds and gaits without central planning by the brain. These observations suggest that a substantial part of leg control in animal and human locomotion is generated by spinal circuits which adapt to changes in the environment [21].

Inspired by these observations on local control, we here present a control for swing leg motions that is based on local feedbacks and does not require predefined swing leg trajectories to robustly place the leg into target points on the ground. First, we develop the control guided by the segment dynamics of a double pendulum (Secs. II \& III). We then test the performance of the control in simulations with a more anthropomorphic swing leg that considers human-like segment mass and inertia distribution as well as the effect of translational hip acceleration (Sec. IV). We find that the identified control can achieve swing leg placement into a large range of target points, that this placement is robust to disturbances in initial conditions and to sudden changes in the target during swing, and that the resulting swing leg trajectories and joint torque patterns compare to those of human walking and running. Finally, we discuss these findings in section $\mathrm{V}$ and summarize our plans for future work.

\section{SWING LEG MODEL}

In human and humanoids, leg placement is largely achieved by the motion of the hip and knee while the ankle contribution can be neglected. To develop intuition about the control strategies for swing leg placement, we use the classic double pendulum with the thigh and shank represented as massless rods of lengths $l_{t}$ and $l_{s}$ with point masses $m_{t}$ and $m_{s}$ attached to their ends (Fig. 1A). The hip is connected to an immovable trunk at the origin of the world frame, and the joint angles $\phi_{h}$ and $\phi_{k}$ are measured as shown in figure 
A

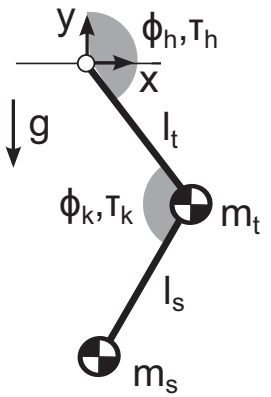

B

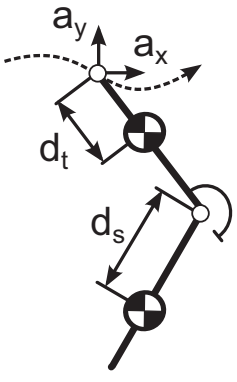

Fig. 1. Swing leg models. (A) Conceptual double pendulum model with point masses at the segment ends. (B) More realistic swing leg model with human-like segment mass distribution and hip translational accelerations as external input.

1A. The resulting equations of motion are

$$
\begin{array}{r}
\left(\left(m_{t}+m_{s}\right) l_{t}-m_{s} l_{s} \cos \phi_{k}\right) l_{t} \ddot{\phi}_{h}= \\
\tau_{h}+\tau_{k}-m_{s} l_{t} l_{s} \cos \phi_{k} \ddot{\phi_{k}}+m_{s} l_{s} l_{t} \sin \phi_{k}\left(\dot{\phi_{k}}-\dot{\phi_{h}}\right)^{2} \\
+\left(m_{t}+m_{s}\right) l_{t} g \sin \phi_{h} \\
m_{s} l_{s}^{2} \ddot{\phi_{k}}= \\
\tau_{k}+m_{s} l_{s}\left(l_{s}-l_{t} \cos \phi_{k}\right) \ddot{\phi}_{h}+m_{s} l_{s} l_{t} \sin \phi_{k} \dot{\phi}_{h}^{2} \\
-m_{s} l_{s} g \sin \left(\phi_{k}-\phi_{h}\right)
\end{array}
$$

for the hip and knee, respectively, where $\tau_{h}$ and $\tau_{k}$ are the applied hip and knee torques.

In addition to the conceptual model, we test the performance of our control with a more realistic simulation model of human swing motions (Fig. 1B). In this second model, the segment inertial properties are based on anthropomorphic data obtained from scaling tables [22] for a human with a body weight and height of $80 \mathrm{~kg}$ and $180 \mathrm{~cm}\left(m_{t}=7 \mathrm{~kg}, m_{s}=4.3 \mathrm{~kg}, d_{t}=21.7 \mathrm{~cm}, d_{s}=30.3 \mathrm{~cm}\right.$, $\left.l_{t}=43 \mathrm{~cm}, l_{s}=43 \mathrm{~cm}\right)$. Moreover, the model includes a knee stop realized by a restoring torque [23]

$\tau_{k}^{r e s}=\left\{\begin{array}{lc}k_{\phi}\left(\phi_{k}-\phi_{\max }\right)\left(1+\dot{\phi}_{k} / \dot{\phi}_{\max }\right), & \dot{\phi}_{k}>-\dot{\phi}_{\max } \\ \phi_{k}>\phi_{\max } \\ 0, & \text { otherwise }\end{array}\right.$

$\left(\phi_{\max }=175 \mathrm{deg}, \dot{\phi}_{\max }=0.01 \mathrm{rads}^{-1}, k_{\phi}=17.2 \mathrm{Nmrad}^{-1}\right)$ and accounts for hip translational accelerations $\left(a_{x}, a_{y}\right)$ in later comparisons to human swing phases in steady walking and running (Sec. IV).

\section{Modular Local Control}

In addition to the fundamental goal of placing the foot into target points, the swing leg motion is subject to the constraint that the foot point needs to clear the ground or an obstacle. We represent both, the placement goal and the clearance constraint, by two control variables, the target leg angle $\alpha_{t g t}$ and the clearance leg length $l_{c l r}$, respectively (Fig. 2A). Assuming equal segment lengths $l_{t}=l_{s}$ (good approximation for human legs) in the remainder of this paper,

the leg angle is given by $\alpha=\phi_{h}-\frac{\phi_{k}}{2}$ and the leg length resolves to $l=2 l_{t} \sin \frac{\phi_{k}}{2}$ (Fig. $2 \mathrm{~B}$ ).

Figure $2 \mathrm{~A}$ outlines a natural sequence of three control tasks which reflects the objective and constraint. First, starting at the ground level from an initial leg angle $\alpha_{0}$, the clearance constraint requires the leg to flex to at least the clearance length $l_{c l r}$ (reached at P). Second, the control focus shifts to advancing the swing leg to the target angle $\alpha_{t g t}$ (reached at Q). And the final task is to extend the leg until ground contact.

Although this sequence of control tasks can be strictly enforced with classical state feedback control, we avoid tracking predefined trajectories for two reasons. Similar to humans [24], we seek to take advantage of the passive dynamics that the swing leg provides to lessen the required torques. In addition, we seek decoupled joint controls as much as possible to modularize the control. Both goals target autonomous and modular replacement legs in rehabilitation robotics. As a consequence, we structure the swing leg control around functionally distinct hip and knee joint controls.

\section{A. Hip Control}

The hip control is active throughout the swing and its primary function is to move the leg into the target angle $\alpha_{t g t}$. The specific control input $\tau_{h}^{\alpha}$ is given by

$$
\tau_{h}^{\alpha}=k_{p}^{\alpha}\left(\alpha_{t g t}-\alpha\right)-k_{d}^{\alpha} \dot{\alpha}
$$

where $k_{p}^{\alpha}$ and $k_{d}^{\alpha}$ are proportional and derivative gains, respectively. Note that a negative input tends to flex the hip. In addition to the angle control, the hip control receives a second input $\tau_{h}^{i i i}$ from the knee control,

$$
\tau_{h}=\tau_{h}^{\alpha}+\tau_{h}^{i i i},
$$

during the last, leg extension stage in the control sequence (after reaching Q). We detail the purpose of $\tau_{h}^{i i i}$ in the next section on knee control. It is the only crossover input between the joints.

\section{B. Knee Control}

The knee control's primary function is to regulate leg length. However, due to the influence of the knee angle on both, leg length and leg angle, the control is more involved. We separate it into the three natural control tasks outlined before (Fig. 2A).

To accomplish the first control task of reaching a minimum clearance $l_{c l r}$ in the initial swing, we take advantage of the passive swing leg dynamics. Equation 2 shows that while the Coriolis, centrifugal and gravitational terms always tend to extend the knee, a negative hip acceleration tends to flex the knee. The hip control (Eq. 4) generates negative hip acceleration initially to drive the leg into the target angle. The resulting passive, negative knee acceleration sufficiently overcomes the opposing Coriolis, centrifugal and gravitational accelerations as long as the leg angle $\alpha$ increases, $\dot{\alpha}>0$ or equivalently $\dot{\phi}_{k}<2 \dot{\phi}_{h}$ (backward motion of foot point). In that case, no input $\tau_{k}$ is required to flex the leg, as the increase in $\alpha$ reinforces hip flexion control and, in turn, 

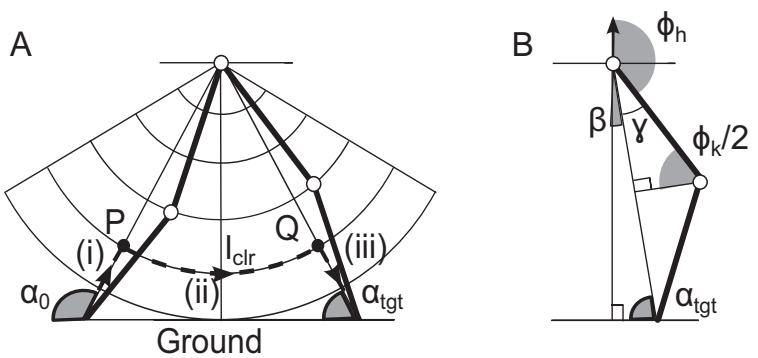

Fig. 2. Swing leg control. (A) Sequence of natural control tasks for reaching a target placement while guaranteeing foot ground clearance. (B) Functional relationship between $\alpha$ and $\phi_{h}$ and $\phi_{k}$. With $\gamma=90-\frac{\phi_{k}}{2}$ and $\beta=$ $180-\gamma-\phi_{h}, \alpha$ relates to $\phi_{h}$ and $\phi_{k}$ as $\alpha=\phi_{h}-\frac{\phi_{k}}{2}$.

passive knee flexion. If on the other hand $\dot{\alpha} \leq 0$, the passive knee flexion is no longer sufficient and the leg tends to scuff the foot. Thus we implement active knee flexion control only in proportion to how fast the leg moves forward,

$$
\tau_{k}^{i}=\left\{\begin{array}{ll}
k^{i} \dot{\alpha}, & \dot{\alpha} \leq 0 \\
0, & \dot{\alpha}>0
\end{array},\right.
$$

where $k^{i}$ is a proportional gain.

Once the leg length has shortened past the clearance length, $l<l_{c l r}$, the knee control switches to the second task of holding the knee so that the leg angle control by the hip can take full effect (Eq. 4). We realize this task with a damping input

$$
\tau_{k}^{i i}= \begin{cases}-k^{i i} \dot{\phi}_{k}, & \dot{\phi}_{k} \leq 0 \\ -k^{i i} \dot{\phi}_{k}\left(\alpha-\alpha_{t g t}\right)\left(\dot{\phi}_{k}+\dot{\alpha}\right), & \dot{\phi}_{k}>0 \& \dot{\phi}_{k}>-\dot{\alpha} \\ 0, & \text { otherwise }\end{cases}
$$

that is pure when the knee flexes $\left(\dot{\phi}_{k} \leq 0\right)$ and modulated when it extends $\left(\dot{\phi}_{k}>0\right)$, with a proportional gain of $k^{i i}$. The implementation avoids the large torques that a precise tracking of $l=l_{c l r}$ would incur fighting the passive swing leg dynamics (Fig. 2A). In particular, when the hip control stops pulling forward, all interaction terms in equation 2 create passive knee extension. The first modulation term $\left(\alpha-\alpha_{t g t}\right)$, enables this passive extension when $\alpha$ approaches its target, easing the third control task. However, when the leg swings faster at higher speeds, passive knee extension amplifies markedly due mainly to the Coriolis acceleration $l_{t} \sin \phi_{k} \dot{\phi}_{h}^{2}$, and the second modulation term $\left(\dot{\phi}_{k}+\dot{\alpha}\right)$, prevents a premature landing of the leg. This term compares the knee extension velocity to the approach velocity $\dot{\alpha}$ (negative when moving forward). If the knee extends faster than the leg moves towards its target angle, damping torque is proportionally activated; otherwise, no control torque is applied.

The final knee control task of stopping swing and extending the leg into the target is initiated when the leg angle passes a threshold $\alpha_{t h r}=\alpha_{t g t}+\Delta \alpha_{t h r}$ and is realized with two functional components. The first component generates a stopping knee-flexion torque

$$
\tau_{k}^{i i i}=\left\{\begin{array}{lc}
-k^{s t p}\left(\alpha_{t h r}-\alpha\right)\left(1-\frac{\dot{\alpha}}{\dot{\alpha}_{\max }}\right), & \quad \alpha<\alpha_{t h r} \\
0, & \quad \text { otherwise }
\end{array}\right.
$$

that is inspired by nonlinear contact models [23], [25], where $k^{s t p}$ is a proportional gain, $\alpha_{t h r}-\alpha$ corresponds to 'ground indentation' and $\dot{\alpha}_{\max }$ is the return velocity at which 'ground resistance' vanishes. The stopping torque works well only if its coupling into the hip motion is canceled and we apply the compensation torque

$$
\tau_{h}^{i i i}=-2 \tau_{k}^{i i i}
$$

at the hip. To motivate this particular compensation, consider equations 1 and 2 at hip and knee angles of $180 \mathrm{deg}$ (vertical leg position). The equations then simplify to

$$
\begin{aligned}
\left(m_{t}+2 m_{s}\right) l_{t}^{2} \ddot{\phi}_{h} & =\tau_{h}+\tau_{k}+m_{s} l_{t}^{2} \ddot{\phi}_{k} \\
m_{s} l_{t}^{2} \ddot{\phi}_{k} & =\tau_{k}+2 m_{s} l_{t}^{2} \ddot{\phi}_{h}
\end{aligned}
$$

where we used the assumption $l_{s}=l_{t}$. Using equation 11 to substitute $\ddot{\phi}_{k}$ in equation 10 , we get

$$
m_{t} l_{t}^{2} \ddot{\phi}_{h}=\tau_{h}+2 \tau_{k}
$$

for the hip equation, which shows that a compensation torque $\tau_{h}=-2 \tau_{k}$ cancels the effect of knee torque on the hip motion.

The second functional component engages when the leg has slowed down to zero angular velocity $\dot{\alpha}=0$, upon which a deliberate extension component is added to the knee torque,

$$
\tau_{k}^{i i i^{\prime}}=\tau_{k}^{i i i}+k^{e x t}\left(l_{0}-l\right),
$$

where $k^{e x t}$ is a proportional gain and $l_{0}=l_{t}+l_{s}$. This active knee extension ensures that the leg reaches down to the ground.

\section{REsults}

The control developed in the previous section is based on the simple double pendulum as a conceptual model (Fig. 1A). However, to test the control performance, we use a swing leg model that considers human-like segment mass distribution and the effect of hip translational accelerations generated by the body (Fig. 1B). With this more realistic model, we first tune the control parameters to achieve robust leg placement into a realistic range of target angles assuming large variations in initial conditions. We then compare the predicted patterns of the foot point motion and the joint torques with those of human walking and running, and evaluate the quality of the leg placement generated by the local feedback control during sudden changes in swing leg targets as well as during horizontal pushes on the foot point that simulate obstacle encounters. 


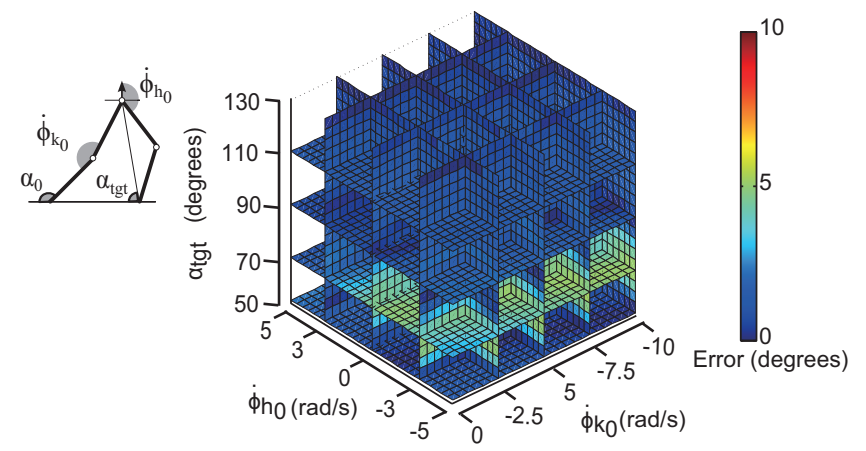

Fig. 3. Swing leg placement into arbitrary targets under large variation in initial conditions. The error $\Delta \alpha=\alpha_{t g t}-\alpha_{l}$ between the target angle and the achieved landing angle is shown as a color code for a large range of initial hip and knee velocities that cover the initial joint velocities observed in human locomotion from $0.65 \mathrm{~ms}^{-1}$ to $5.5 \mathrm{~ms}^{-1}$.

\section{TABLE I}

CONTROL PARAMETERS VALUES. VALUES IN PARENTHESES ARE GAINS ADAPTED FOR WALKING \& RUNNING RESPECTIVELY.

\begin{tabular}{c|c||c|c} 
parameter & value & parameter & value \\
\hline$k_{p}^{\alpha}$ & $110(50 / 150) \mathrm{Nmrad}^{-1}$ & $k^{i}$ & $13 \mathrm{Nmsrad}^{-1}$ \\
$k_{d}^{\alpha}$ & $8.5(5.5 / 8.5) \mathrm{Nmsrad}^{-1}$ & $k^{i i}$ & $5.5 \mathrm{Nmsrad}^{-1}$ \\
$k^{\text {stp }}$ & $250(60 / 160) \mathrm{Nmrad}^{-1}$ & $\dot{\alpha}_{\max }$ & $10 \mathrm{rads}^{-1}$ \\
$k^{\text {ext }}$ & $200 \mathrm{~N}$ & $\alpha_{t h r}$ & $\alpha_{\text {tgt }}+8 \mathrm{deg}$
\end{tabular}

\section{A. Leg placement into arbitrary targets}

The initial leg configuration is set to $\phi_{h, 0}=220 \mathrm{deg}$ and $\phi_{k, 0}=175 \mathrm{deg}\left(\alpha_{0}=132.5 \mathrm{deg}\right)$, the clearance leg length is fixed at $l_{c l r}=5 \mathrm{~cm}$, and the control gains of the local feedback control are hand-tuned with the goal of minimizing the error between the target leg angle and the actual landing angle for a large range of initial joint velocities and target angles. Simultaneously, peak joint torques are tuned to be within the peak torques observed in human walking and running. Note that the hip is still assumed to be hinged during tuning as it is unclear what external translational accelerations should be applied in this process. The resulting set of control parameters is shown in table I. Figure 3 shows the resulting control performance for target angles $\alpha_{t g t}=$ $50 \ldots 130 \mathrm{deg}$ that include typical human landing leg angles and for initial joint velocities $\dot{\phi}_{h, 0}=-5 \ldots 5 \mathrm{rads}^{-1}$ and $\dot{\phi}_{k, 0}=-10 \ldots 0 \mathrm{rads}^{-1}$ that cover the experimental data on initial joint velocities in human locomotion $\left(\dot{\phi}_{h, 0}=-4 \ldots 2\right.$ $\operatorname{rads}^{-1}$ and $\dot{\phi}_{k, 0}=-7 \ldots-1 \mathrm{rads}^{-1}$, see section IV-B for details on experimental data). The identified feedback control can achieve low target placement errors with an average error of $1.4 \mathrm{deg}$ and a maximum error of $5.2 \mathrm{deg}$ throughout the large ranges of target angles and initial joint velocities. Similar results hold for different ground clearance constraints and initial leg configurations $\left(l_{c l r}=5,8 . . \mathrm{cm}\right.$ and $\alpha_{0}=132,140$..deg).

\section{B. Comparison to human walking and running}

For the comparison to human swing motions, we recorded multiple swing leg trajectories of a subject walking from $0.65 \mathrm{~ms}^{-1}$ and $2 \mathrm{~ms}^{-1}$ and running from $2.5 \mathrm{~ms}^{-1}$ to $5.5 \mathrm{~ms}^{-1}$,
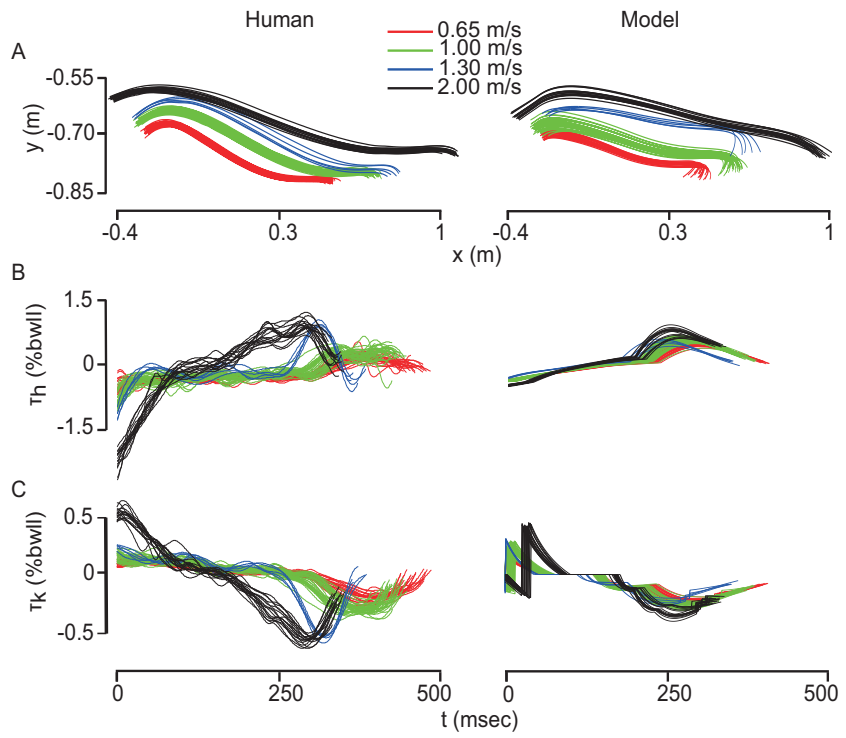

Fig. 4. Observed and predicted swing leg patterns in walking, respectively. The ankle trajectories (A), hip and knee torques (B\&C) during multiple swing phases (individual traces) are compared between human walking and model prediction at four different walking speeds (color coded). For the model, the initial joint positions and speeds as well as the hip translational accelerations are taken from the corresponding human swing motions. The coordinates of the foot point in A are given with respect to a world frame that originates at the initial position of the hip. The torques in $\mathrm{B}$ and $\mathrm{C}$ are normalized to percent of body weight times leg length (\%bwll).

covering slow to fast walking as well as slow to fast running. The left columns in figures 4 and 5 show the ankle trajectories (A) and the corresponding hip and knee torques (B and $\mathrm{C}$, obtained from inverse dynamics) observed during human swing in walking and running at the different speeds.

The model-predicted swing leg patterns are similar to the observed ones in walking. The right column in figure 4 shows the model-predicted swing phase motion of the foot point (tip of shank segment) and the corresponding joint torque patterns. (For all speeds, the model's target angle is $\alpha_{t g t}=70 \mathrm{deg}$, which equals the observed landing angle $\alpha_{l}=70 \pm 3$ deg.) Also, hip translational accelerations are applied in the model based on the observed hip accelerations for each swing phase. As a result, the hip control gains had to be adjusted to one set of lower values for all walking trials and of higher values for all running trials, Tab. I) The foot point motion has a similar range and shape for all four walking speeds (A), and the hip and knee torques have similar magnitudes and gross behavior (B and $\mathrm{C}$ ). In the model, the hip torque pattern is generated by the target angle control (Eq. 4) throughout swing, except for the sharp rise in hip extension torque which appears in the second half of swing due to the knee compensation $\tau_{h}^{i i i}=-2 \tau_{k}^{i i i}$ (Eqs. 5 and 9). This sharp rise also occurs in the knee torque pattern and is generated by the knee stopping torque (Eq. 8). One marked difference in the knee torque pattern appears at $2.00 \mathrm{~ms}^{-1}$ walking, where, before the knee damping occurs that prevents leg flexion past the clearance length (Eq. 7), the model shows an initial knee flexion torque to actively 

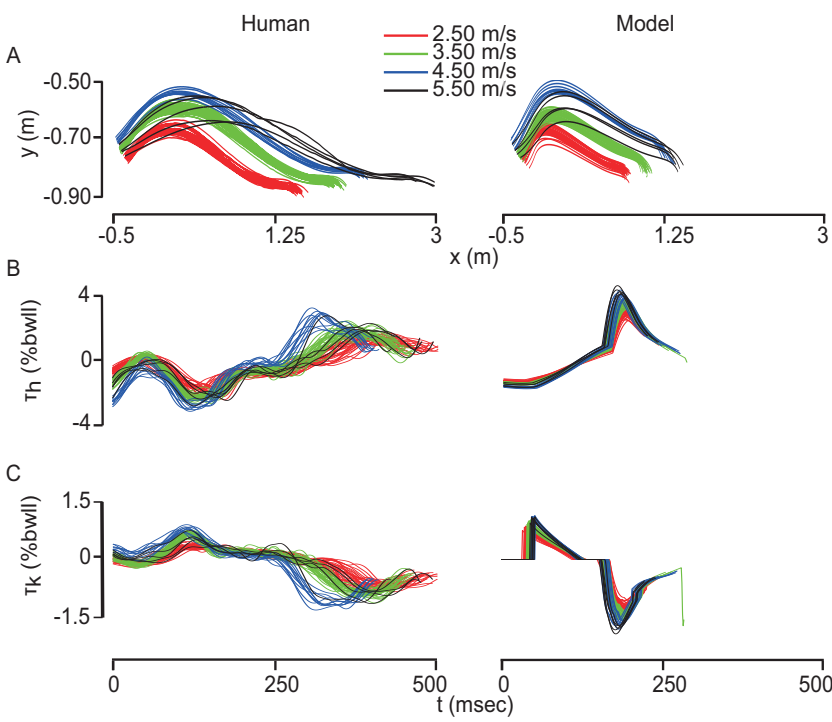

Fig. 5. Observed and predicted swing leg patterns in running, respectively See caption of figure 4 for explanations. Note that for only one swing at $3.5 \mathrm{~ms}^{-1}$, the model engaged knee stop (knee torque spike at end.)

prevent scuffing the foot (Eq. 6). This initial flexion torque is not visible in human walking. The difference is related to the much larger initial hip flexion torque that humans use at this speed, which passively flexes the knee and thus prevents foot scuffing. It points to a speed-dependent increase in the hip control gains not included in the model.

In contrast to walking, the predicted patterns for running have a shorter stride length and a clearly faster swing time than present in human running (Fig. 5). Despite these differences, the shapes of the torque patterns match human data in general (B and $\mathrm{C}$ ). The shape of the hip torque pattern is similar between walking and running in the model. Except for an initial modulation, this general shape is also preserved in the human hip torque pattern between walking and running. By contrast, the knee torque pattern shows an added, initial period of silence in running which is present in both the model and the human data. This silent period occurs in the model as the initial knee angle in the swing phase of running $\left(\phi_{k, 0}=149 \pm 2.5 \mathrm{deg}\right)$ is more extended than in walking $\left(\phi_{k, 0}=120 \pm 2 \mathrm{deg}\right)$, and the knee passively flexes before the leg reaches the clearance length where the damping torque engages (Eq. 7).

The short swing phase in running is caused mainly by an increased proportional gain $k_{p}^{\alpha}$ for the leg angle control at the hip (Eq. 4 and Tab. I). We required this modification in the model, because otherwise the passive leg flexion by the hip control is too weak and the large hip translational accelerations in running force the leg into an early landing. The modification also entails a larger gain $k^{\text {stp }}$ for the stopping control (Eq. 8 and Tab. I).

\section{Reaction to target changes and obstacle encounters}

In addition to comparing the predicted swing leg patterns to human swing motions, we test the performance of the control in the presence of disturbances. First, we consider
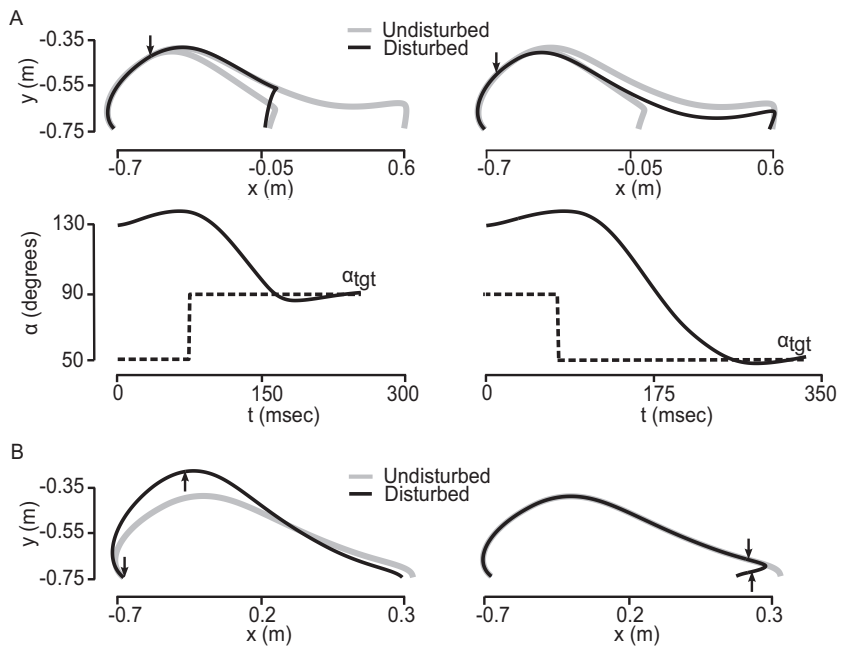

Fig. 6. Reaction to disturbances during swing. The reaction to disturbances during swing is shown for large changes in the target leg angle $\alpha$ and during simulated obstacle encounters for characteristic swing phases with initial conditions that match human walking at $1.3 \mathrm{~ms}^{-1}$. Undisturbed swing phases are shown in gray and disturbed ones in black. (A) The target angle (dashed line) is changed at $75 \mathrm{~ms}$ into the swing from $50 \mathrm{deg}$ to $90 \mathrm{deg}$ (left column) and vice versa (right column). The arrows mark the foot point position at the time of change. (B) An external impulse of $15 \mathrm{Ns}$ is applied to the foot point in early (left) and late swing (right), resulting in an elevated foot point trajectory and premature landing, respectively. The arrows indicate the start and end of the force application.

sudden changes in the target angle. Figure 6A shows the characteristic response of the model to changes in the target angle from $\alpha_{t g t}=50 \mathrm{deg}$ to $\alpha_{t g t}=90 \mathrm{deg}$ and vice versa (left and right columns, respectively), which are initiated at $75 \mathrm{~ms}$ into the swing phase. In both cases, the control rapidly adapts and guides the foot point into the new target position with negligible error. We observe a similar performance for the same changes initiated later in the swing (not shown), up to $150 \mathrm{~ms}$ from $50 \mathrm{deg}$ to $90 \mathrm{deg}$ (at which the leg angle crosses $\alpha=90 \mathrm{deg}$ in the undisturbed swing) and up to $225 \mathrm{~ms}$ for the reverse change (which equals about $80 \%$ of the swing time of its corresponding undisturbed case).

Figure $6 \mathrm{~B}$ shows the characteristic response of the model to an impulse disturbance of $15 \mathrm{Ns}$ in early and late swing (left and right columns). The impulse simulates obstacle encounters and is applied as a constant force applied to the foot point in the negative $\mathrm{x}$-direction. The model shows an elevating response for the early disturbance and a premature landing for the late disturbance. Both responses are observed in human locomotion and often assumed to reflect two explicit control strategies [26]. By contrast, the model shows these two behaviors as inherent outcomes of its placement control.

\section{CONCLUSion AND Future Work}

We developed a control for swing leg placement. The control is structured around a sequence of three tasks that include flexing the leg into a clearance length, advancing the leg into a target angle, and extending it until ground contact (Fig. 2). Although this sequence can be enforced with strict 
trajectory tracking, we target energy efficient and modular artificial legs in rehabilitation robotics and developed the control to take advantage of passive swing leg dynamics and to modularize the individual hip and knee controls.

Our results suggest that the identified control can achieve swing leg placement into a large range of targets for a wide range of walking and running speeds with robustness to disturbances in initial conditions and to sudden changes in the placement target during swing. In the simulation model, the control achieves leg placement into target angles ranging from $50 \mathrm{deg}$ to $130 \mathrm{deg}$ with an average error of $1.4 \mathrm{deg}$ and a maximum error of $5.2 \mathrm{deg}$ throughout a large range of initial joint velocities (Fig. 3). This accuracy is maintained even when the target angles are suddenly changed during swing (Fig 6A). This performance is in stark contrast to current control approaches using trajectory tracking, which need to retune trajectories for different locomotion speeds and to provide explicit compensation trajectories when encountering disturbances [18].

In addition, the control generates swing patterns that share the main features of human swing leg patterns. In undisturbed swings, the control generates foot point and joint torque patterns that are similar to those patterns observed in human walking and running (Figs. 4 and 5). One major difference is the substantially shorter swing time in running in the model, which coincides with clearly shorter stride lengths. During simulated obstacle encounters in early and late swing, the model generates an elevating response and a premature landing, respectively (Fig 6B). Both responses are well known behaviors in disturbed human swing phases [26]. In contrast to generating these responses with explicitly triggered control [18], [27], they are inherent to the identified leg placement control.

In future work, we plan to overcome the timing difference in the swing leg control by incorporating the effects of the hip translational acceleration and to map the identified control functions onto neuromuscular structure present in the human leg. This step will enable indirect verification of the control by comparing predicted and observed muscle actuations in disturbed human swing phases. In addition, we plan to transfer the control to robotic legs in humanoid and rehabilitation robotics, for which we are currently developing a robotic leg testbed [28].

\section{ACKNOWLEDGMENT}

This work is supported by the NSF through the NSF ERC on Quality of Life Technology (EEC 0540865).

\section{REFERENCES}

[1] M. Raibert, Legged robots that balance. MIT press, Cambridge, 1986.

[2] M. A. Townsend, "Biped gait stabilization via foot placement," $J$ Biomech, vol. 18, no. 1, pp. 21-38, 1985.

[3] S. Kajita, F. Kanehiro, K. Kaneko, K. Yokoi, and H. Hirukawa, "The $3 \mathrm{~d}$ linear inverted pendulum mode: a simple modeling for a biped walking pattern generation," in IEEE/RSJ International Conference on Intelligent Robots and Systems, vol. 1, 2001, pp. 239 -246 vol.1.

[4] R. Blickhan, "The spring-mass model for running and hopping," J. of Biomech., vol. 22, pp. 1217-1227, 1989.

[5] T. McMahon and G. Cheng, "The mechanism of running: how does stiffness couple with speed?" J. of Biomech., vol. 23, pp. 65-78, 1990.
[6] J. Hodgins and M. N. Raibert, "Adjusting step length for rough terrain locomotion," IEEE Trans on Robotics and Automation, vol. 7, no. 3, pp. 289-298, 1991.

[7] J. Pratt, J. Carff, S. Drakunov, and A. Goswami, "Capture point: A step toward humanoid push recovery," in Proceedings of the 6th IEEE-rAS Intl Conf on Humanoid Robots, 2006.

[8] F. Parietti and H. Geyer, "Reactive balance control in walking based on a bipedal linear inverted pendulum model," in IEEE Int Conf on Robotics and Automation, 2011, pp. 5442-5447.

[9] A. Wu and H. Geyer, "The 3d spring-mass model reveals a time-based deadbeat control for highly robust running and steering in uncertain environments," IEEE Trans on Robotics, submitted.

[10] J. Morimoto, G. Zeglin, and C. G. Atkeson, "Minimax differential dynamic programming: Application to a biped walking robot," in IEEE/RSJ Int. Conf. on Intelligent Robots and Systems, 2003, pp. 1927-1932.

[11] P. Michel, J. Chestnutt, S. Kagami, N. Koichi, J. Kuffner, and T. Kanade, "Gpu-accelerated real-time 3d tracking for humanoid locomotion and stair climbing," in IEEE/RSJ Int. Conf. on Intelligent Robots and Systems, 2007, pp. 463-469.

[12] M. Kanako, M. Mitsuharu, F. Kanehiro, S. Kajita, K. Kaneko, and K. Yokoi, "Human-like walking with toe supporting for humanoids," in IEEE/RSJ Int. Conf. on Intelligent Robots and Systems, 2011, pp. $4428-4435$.

[13] D. L. Grimes, "An active multi-mode above knee prosthesis controller," $\mathrm{PhD}$ thesis, Department of Mechanical Engineering, Massachusetts Institute of Technology, 1979.

[14] J. L. Johansson, D. M. Sherrill, P. O. Riley, P. Bonato, and H. Herr, "A clinical comparison of variable-damping and mechanically passive prosthetic knee devices," American Journal of Physical Medicine and Rehabilitation, vol. 84, no. 8, pp. 563-575, 2005.

[15] S. K. Au, H. Herr, J. Weber, and E. C. Martinez-Villalpando, "Powered ankle-foot prosthesis for the improvement of amputee ambulation," Conf Proc IEEE Eng Med Biol Soc, vol. 2007, pp. 3020-6, 2007.

[16] K. Fite, J. Mitchell, F. Sup, and M. Goldfarb, "Design and control of an electrically powered knee prosthesis," in Proc IEEE Int Conf on Rehabilitation Robotics, 2007, pp. 902-905.

[17] M. Holgate, A. Bohler, and T. Sugar, "Control algorithms for ankle robots: A reflection on the state-of-the-art and presentation of two novel algorithms," in 2nd IEEE RAS EMBS International Conference on Biomedical Robotics and Biomechatronics, 2008, pp. 97 -102.

[18] B. E. Lawson, H. A. Varol, F. Sup, and M. Goldfarb, "Stumble detection and classification for an intelligent transfemoral prosthesis," in Annual Int Conf of IEEE EMBS, 2010, pp. 511-514.

[19] M. L. Shik, F. V. Severin, and G. N. Orlovski, "Control of walking and running by means of electric stimulation of the midbrain," Biofizika, vol. 11, no. 4, pp. 659-666, 1966.

[20] G. Courtine, Y. Gerasimenko, R. van den Brand, A. Yew, P. Musienko, H. Zhong, B. Song, Y. Ao, R. M. Ichiyama, I. Lavrov, R. R. Roy, M. V. Sofroniew, and V. R. Edgerton, "Transformation of nonfunctional spinal circuits into functional states after the loss of brain input," Nat Neurosci, vol. 12, no. 10, pp. 1333-42, 2009.

[21] H. Hultborn and J. B. Nielsen, "Spinal control of locomotion - from cat to man," Acta Physiologica, vol. 189, no. 2, pp. 111-121, 2007.

[22] D. A. Winter, Biomechanics and motor control of human movement, 4th ed. Hoboken, N.J.: Wiley, 2009.

[23] H. Geyer and H. Herr, "A muscle-reflex model that encodes principles of legged mechanics produces human walking dynamics and muscle activities," IEEE Trans Neural Syst Rehabil Eng, p. DOI 10.1109/TNSRE.2010.2047592, 2010.

[24] S. Mochon and T. McMahon, "Ballistic walking," J. Biomech., vol. 13, no. 1 , pp. 49-57, 1980

[25] K. Hunt and F. Crossley, "Coefficient of restitution interpreted as damping in vibroimpact," Journal of Applied Mechanics, vol. 42, no. 2, pp. $440-445,1975$.

[26] J. J. Eng, D. A. Winter, and A. E. Patla, "Strategies for recovery from a trip in early and late swing during human walking," Exp Brain Res, vol. 102 , no. 2, pp. 339-49, 1994.

[27] T. Shiratori, B. Coley, R. Cham, and J. K. Hodgins, "Simulating balance recovery responses to trips based on biomechanical principles," in Proceedings of the 2009 ACM SIGGRAPH/Eurographics Symposium on Computer Animation, ser. SCA '09. ACM, 2009, pp. 37-46.

[28] A. Schepelmann, M. Taylor, and H. Geyer, "Development of a testbed for robotic neuromuscular controllers," in Proceedings of Robotics: Science and Systems, 2012. 